\title{
Intuitionistic Fuzzy Multiple Model Tracking Algorithm for Tracking Manoeuvring Target
}

\author{
Renhou Zhao, Xiaoguang Zhou \\ Flight Simulation Training Department \\ Naval Aviation Institute \\ Hu Ludao, LiaoNing,125001,China \\ zhanglili2117@163.com
}

\author{
Lili Zhang \\ Institute College of Opto-electronic Engineering \\ Changchun University of Science and Technology \\ ChangChun 130022, China \\ zhangliligf@163.com
}

\begin{abstract}
A tracking algorithm for maneuvering target based on intuitionistic logic inference is developed in this paper. Firstly, in order to simply the calculation, the filtering measurement innovations tacked with the innovation covariance are used as the inputs for the intuitionistic fuzzy inference system to get the matched degrees for each filtering model in the model set designed, which is to in replace of the model probability computed intricately in IMM. Secondly, by transferring the uncertainty from measurement space to intuitionistic fuzzy space, IFMMTA has improved the precision of mode inference. And at the same time, combining the intuitionistic fuzzy inference with EKF and adopting parallel dealing method makes IFMMTA more efficiency for maneuvering target real-time tracking. In the end, the performance of IFMMAT is tested via Monte Carlo simulation, which indicates that IFMMAT is better than FMMTA to reduce the position and velocity tracking error for the maneuvering target.
\end{abstract}

Keywords-maneuvering target tracking; Intuitionnistic fuzzy multiple model

\section{INTRODUCTION}

The prevailing approaches to maneuvering target tracking are the modeling of the target motion/dynamics to cover the unknown modeling errors or deviations of the model from the true system. The so-called multiple-model (MM) method is a major approach, which is also probably the most natural approach to solve the hybrid estimation [1][2]. The paper [3] presents an algorithm (FMMTA) for a maneuvering tracking based on fuzzy logic inference. FMMTA enhances the adaptiveness of MM though mapping the uncertainty of measurement space to fuzzy space, and the uncertainly of measurement space is resolved via the fuzzy inference mechanism. In real-life situations, when a person is asked to express his/her preference degree to an object, there usually exists an uncertainty or hesitation about the degree, and there is no means to incorporate the uncertainty or hesitation in a fuzzy set. To solve this issue, Atanassov generalized Zadeh's fuzzy set to intuitionistic fuzzy set (IFS) by adding an uncertainty (or hesitation) degree, IFS is more suitable to describe the fuzzy essence of world[4][5][6]. So a new tracking algorithm is put forward based on intuitionistic fuzzy logic inference for maneuvering target, called the intuitionistic fuzzy multiple model tracking algorithm (IFMMTA). Section II predicates calculus on Intuitionistic Fuzzy Logic (IFL), a symmetric synthetic method for finding the truth of IFL propositions using membership and hesitancy degree is proposed on the basis of the hypothesis of an equilibrium state of supportability and opposability of neutral evidences indicated in the intuitionistic index. The fundamental operation rules of IFL propositions are presented. The investigation is with an emphasis on the techniques for multi-dimensions conditional reasoning on IFL. Section III provides the models for manoeuvring target tracking and the IMM briefly, and IFMATA is designed in Section IV. Then the performance of IFMMTA is given in section while comparing with FMATA and IMM, Finally a conclusion is presented in section VI.

\section{INTUITIONISTIC FUZZY LOGIC INFERENCE}

The basic notions of IFSs that are going to be used throughout the paper are briefly described.

Definition 1: An intuitionistic fuzzy set $A$ defined on a universe $X$ is given by [7]

$$
A=\left\{\left\langle x, \mu_{A}(x), v(x)\right\rangle \mid x \in X\right\}
$$

Where

$$
\mu_{A}(x): X \rightarrow[0,1] \text { and } v(x): X \rightarrow[0,1]
$$

With the condition

$$
0 \leq \mu_{A}(x)+v(x) \leq 1
$$

For all $x \in X$.

For an IFS $A$ in $X$ the $\pi_{A}(x)$ is called intuitionistic index of an element $x \in X$ in $A$ following expression:

$$
\pi_{A}(x)=1-\mu_{A}(x)-v_{A}(x)
$$

The $\pi_{A}(x)$ is considered as the hesitancy degree of $x$ to A.

Intuitionistic fuzzy is the extension and development of fuzzy set theory, so are intuionistic fuzzy proposition and logic to fuzzy proposition and logic[7][8]. Intuionistic fuzzy proposition $P$ is a language statement of notion that without definitude limit, it can express people's subjective thought, but the subjective thought that varies between different 
people. Truth-value of intuionistic fuzzy proposition $P$ is any value in $[0,1]$, the process of evaluation is a mapping $T: u \in U \rightarrow\{0,1\}$ from [0,1] to intuionistic fuzzy proposition set $U$.If intuionistic fuzzy proposition $P$ is corresponding to intuionistic fuzzy $A$, then the truth-value of $P$ is defined as:

$$
T(P)=\alpha\left[\mu_{A}(x)+\beta \cdot \pi_{A}(x)\right]
$$

Where $\alpha, \beta$ is the composed symmetry coefficient of degree of membership and degree of hesitancy of $x \in A$. In a nutshell, let $\alpha=1$ and $\beta=0.5$, and then

$$
T(P)=\mu_{A}(x)+\frac{1}{2} \pi_{A}(x)
$$

So the Truth-value of $P$ is equal to the composing of degree of membership and degree of non-membership of $x \in A$. The meaning of $\beta=0.5$ is that the intuitionistic fuzzy index is neutrality, the degree of approve and averse is in equilibrium state.

The main formats of Intuionistic fuzzy reasoning are implications, conditions, multiplications, multi-dimensions. In multi-model tracking algorithm, multi-dimensions reasoning is mainly used, multi-dimensions reasoning is defined:

If $A_{i}, B_{i} \in[0,1]$ is an intuitionistic fuzzy proposition, and $A_{i}$ gets vale on discuss field $X, B_{i}$ gets vale on discuss field $X$, the relation of $A_{i} \rightarrow B_{i}$ is $R_{i}$, $i=1, \cdots, n$, then $A_{1} \rightarrow B_{1}, A_{2} \rightarrow B_{2}, \cdots, A_{n} \rightarrow B_{n}$ is an intuitionistic fuzzy multi-dimensions reasoning, a compose relation of $R\left(A_{1} \rightarrow B_{1}, A_{2} \rightarrow B_{2}, \cdots, A_{n} \rightarrow B_{n}\right)$ is defined as :

$$
\begin{aligned}
R\left(A_{1}\right. & \left.\rightarrow B_{1}, A_{2} \rightarrow B_{2}, \cdots, A_{n} \rightarrow B_{n}\right)=\bigcup_{i=1}^{n} R_{i}\left(A_{i} \rightarrow B_{i}\right) \\
& =\int_{X \times Y}\left\langle\mu_{A_{1} \rightarrow B_{1}, A_{2} \rightarrow B_{2}, \cdots, A_{n} \rightarrow B_{n}}(x, y), \gamma_{A_{1} \rightarrow B_{1}, A_{2} \rightarrow B_{2}, \cdots, A_{n} \rightarrow B_{n}}(x, y)\right\rangle /(x, y)
\end{aligned}
$$

where:

$$
\begin{aligned}
\mu_{A_{1} \rightarrow B_{i}, A_{2} \rightarrow B_{2}, \cdots, A_{n} \rightarrow B_{n}}(x, y) & ={ }_{i=1}^{n}\left(\mu_{A_{i}}(x) \wedge \mu_{B_{i}}(y)\right) \\
= & \left(\mu_{A_{i}}(x) \wedge \mu_{B_{i}}(x)\right) \vee\left(\mu_{A_{i}}(x) \wedge \mu_{B_{i}}(x)\right) \vee \cdots \vee\left(\mu_{A_{i}}(x) \wedge \mu_{B_{n}}(x)\right)
\end{aligned}
$$$$
\gamma_{A_{1} \rightarrow B_{1}, A_{2} \rightarrow B_{2}, \cdots, A_{n} \rightarrow B_{n}}(x, y)=\hat{i=1}_{i=1}^{n}\left(\gamma_{A_{i}}(x) \vee \gamma_{B_{i}}(y)\right)
$$$$
=\left(\gamma_{A_{1}}(x) \vee \gamma_{B_{1}}(x)\right) \wedge\left(\gamma_{A_{1}}(x) \vee \gamma_{B_{1}}(x)\right) \wedge \cdots \wedge\left(\gamma_{A_{n}}(x) \vee \gamma_{B_{n}}(x)\right)
$$

The truth-value of $A_{i} \rightarrow B_{i} i=1, \cdots, n$ is

$$
T\left(A_{1} \rightarrow B_{1}, A_{2} \rightarrow B_{2}, \cdots, A_{n} \rightarrow B_{n}\right)=\underset{i=1}{\vee}\left(T\left(A_{i}\right) \wedge T\left(B_{i}\right)\right)
$$

If $A^{*}$ and $R_{A_{1} \rightarrow B_{1}, A_{2} \rightarrow B_{2}, \cdots, A_{n} \rightarrow B_{n}}$ are known, we can $B^{*}$ from the composed operation:

$$
B^{*}=A^{*} \circ R_{A_{1} \rightarrow B_{1}, A_{2} \rightarrow B_{2}, \cdots, A_{n} \rightarrow B_{n}}
$$

On the other hand, if $B^{*}$ and $R_{A_{1} \rightarrow B_{1}, A_{2} \rightarrow B_{2}, \cdots, A_{n} \rightarrow B_{n}}$ are known, we can $A^{*}$ from the composed operation:

$$
A^{*}=R_{A_{1} \rightarrow B_{1}, A_{2} \rightarrow B_{2}, \cdots, A_{n} \rightarrow B_{n}} \circ B^{*}
$$

\section{MATHEMATICS MODELS OF TRACKING MANOEUVERING TARGET}

It is supposed that motion state of target and observation can be described by mathematical model, a class of stochastic hybrid system with additive noise is used to discrete dynamic model of manoeuvering target :

$$
\begin{gathered}
x(k+1)=F_{k}[k, x(k), u(k)]+\omega(k) \\
z(k)=H_{k}[x(k)]+v(k)
\end{gathered}
$$

Where $x(k)$ is the base state vector of moving target at time $k, u(x)$ is known input vector, $\omega(k)$ and $v(k)$ are the process and measurement noise sequences, respectively. It is assumed that the process and measurement noise are independent with each other, and are white with mean of $\bar{\omega}(k), \bar{v}(k)$, covariances of $Q(k)$ and $R(k)$ respectively. It is obvious that the plant is a nonlinear system, where $x(k)$ and $z(k)$ depend on the uncertain mode of moving target.

For a target manoeuvres in the $x-y$ plane in uniform velocity, its motion can be described by two orders constant velocity model[2]:

$$
x(k+1)=\left[\begin{array}{cccc}
1 & T & 0 & 0 \\
0 & 1 & 0 & 0 \\
0 & 0 & 1 & T \\
0 & 0 & 0 & 1
\end{array}\right] x(k)+\left[\begin{array}{cc}
\frac{1}{2} T^{2} & 0 \\
T & 0 \\
0 & \frac{1}{2} T^{2} \\
0 & T
\end{array}\right] v(k)
$$

It is supposed the radar only measures position information in $x-y$ plane, then the measure equation is define as:

$$
z(k+1)=\left[\begin{array}{cccc}
1 & 0 & 0 & 0 \\
0 & 0 & 1 & 0
\end{array}\right] x(k+1)+\omega(k+1)
$$

Where $T$ is sampling time, $x=[\varepsilon \dot{\varepsilon} \eta \dot{\eta}]$ is the state variable of the target, $\varepsilon$ and $\eta$ is position in the $x-y$ plane, $\dot{\varepsilon}$ and $\dot{\eta}$ is velocity. Because of environment interference, the target doesn't do exactly uniform velocity, there is a small acceleration. Gauss noise $v$ approximately describes acceleration with covariance $Q(k)$. 
If the target does swerve motion, the motion can be described by linkage swerve model:

$$
\begin{aligned}
x(k) & =\left[\begin{array}{ccccc}
1 & \frac{\sin (\omega T)}{\omega} & 0 & \frac{1-\cos (\omega T)}{\omega} & 0 \\
0 & \cos (\omega T) & 0 & -\sin (\omega T) & 0 \\
0 & \frac{1-\cos (\omega T)}{\omega} & 1 & \frac{\sin (\omega T)}{\omega} & 0 \\
0 & \sin (\omega T) & 0 & \cos (\omega T) & 0 \\
0 & 0 & 0 & 0 & 1
\end{array}\right] x(k) \\
+ & {\left[\begin{array}{ccc}
\frac{1}{2} T^{2} & 0 & 0 \\
T & 0 & 0 \\
0 & \frac{1}{2} T^{2} & 0 \\
0 & T & 0 \\
0 & 0 & T
\end{array}\right] v(k) }
\end{aligned}
$$

The measurement equation is defined as:

$$
z(k+1)=\left[\begin{array}{lllll}
1 & 0 & 0 & 0 & 0 \\
0 & 0 & 1 & 0 & 0
\end{array}\right] x(k+1)+\omega(k+1)
$$

Where $\omega$ is the angle velocity. Because of angle velocity $\omega$, the state variable is extended as $x=[\varepsilon \dot{\varepsilon} \eta \dot{\eta} \omega]$. Because the formula (14) is nonlinear, so the EKF is adopted to be used.

\section{THE DESIGN FOR IFMMTA ALGORITHM}

\section{A. Model filtering}

According to the measurement input $z(k)$ from sensor for maneuvering target, the $i$ th sub-model filtering outputs is calculated as following:

$$
\begin{gathered}
\widehat{X}_{i}(k+1 \mid k)=F_{i}(k) \hat{X}_{i}(k)+\Gamma_{i}(k) U_{i}(k) \\
\tilde{X}_{i}(k+1 \mid k)=F_{i}(k) \tilde{X}_{i}(k)+G_{i}(k) W_{i}(k) \\
P_{i}(k+1 \mid k)=F_{i}(k) P_{i}(k \mid k) F_{i}^{T}(k)+Q_{i}(k) \\
\hat{Z}_{i}=(k+1 \mid k)=H_{i}(k+1) \hat{X}_{i}(k+1 \mid k) \\
\tilde{Z}_{i}(k+1 \mid k)=H_{i}(k+1) \tilde{X}_{i}(k+1 \mid k)+V_{i}(k+1) \\
S_{i}(k+1)=H_{i}(k+1) P(k+1 \mid k) H_{i}^{T}(k+1)+R_{i}(k+1)(20) \\
K_{i}(k+1)=P_{i}(k+1 \mid k) H_{i}^{T}(k+1) S_{i}^{-1}(k+1) \\
\hat{X}_{i}(k+1 \mid k+1)=\hat{X}_{i}(k+1 \mid k)+K_{i}(k+1) \tilde{Z}_{i}(k+1 \mid k)(22) \\
P_{i}(k+1 \mid k+1)=P_{i}(k+1 \mid k)-K_{i}(k+1) S_{i}(k+1) K_{i}^{T}(k+1)(23)
\end{gathered}
$$

According to result of the above sub-model filtering, the state estimation and covariance for tracking target to model $i$ is taken, and also the measurement innovation $\tilde{z}_{i}$ and its error covariance $S_{i}$ to the model $i$ can be computed. Therefore the crisp input to intuitionistic inference system is denoted by the following:

$$
E_{i}=\left(\tilde{z}_{i}\right)^{T}\left(S_{i}\right)^{-1} \tilde{z}_{i} i \in N
$$

From the stochastic theory, we know that the distribution of $E_{i}$ is $\chi^{2}$, which will be available when designing the member and non-membership functions later.

\section{B. Intuitionistic fuzzy reasoning system}

Assuming that the universe discourse for the crisp input $E_{i}$ is $A_{i}, \tilde{E}_{i}$ is the intuitionistic fuzzy value $E_{i}$, and assuming that the different sub-filtering model has the same unversed discourse:

$$
A_{i}=A_{j}, i, j \in M, i \neq j
$$

Defines the similar intuitionistc fuzzy subsets to the crisp input space: biggest ( $B T$ ) , bigger $(B R)$, big ( $B$ ), middle $(M)$, small $(S), \operatorname{smaller}(S R)$, smallest $(S T)$. The Gauss function is selected as the fuzzy membership function of $E_{i}$ :

$$
\mu(x)=\exp \left(\frac{-(x-c)^{2}}{2 \sigma^{2}}\right)
$$

The non-membership functions defined as:

$$
\gamma(x)=\left(1-\exp \left(\frac{-(x-c)^{2}}{2 \sigma^{2}}\right)\right)^{k}, k \geq 1
$$

Where $c 、 \sigma 、 k$ are coefficient, which need to be designed. Assuming that the output space intuitionistic fuzzy inference system is $[a, b], 0 \leq a \leq 1,0 \leq b \leq 1, a+b \leq 1$, and defines the intuitionistic fuzzy subsets as: biggest $(B T)$, bigger $(B R)$, big $(B)$, middle $(M)$, small $(S)$, smaller( $S R$ ), smallest $(S T$ ),similar to the above, but the membership and non-membership function is triangular forms. Then according to the characteristics of intuitionistic inference system, the intuitionistic linguistic rules are as following:

Rule1:

if $\tilde{E}_{1}=B T$ and $\tilde{E}_{2}=B T \cdots \widetilde{E}_{i}=S T \cdots \widetilde{E}_{N}=B T$ then $\tilde{\mu}_{1}=S T$ and $\tilde{\mu}_{2}=S T \cdots \tilde{\mu}_{i}=B T \cdots \tilde{\mu}_{N}=S T$

\section{C. $\quad$ The matched degree $\mu_{i}$ for model $i$}

According to intuitionistic linguistic rules, the intuitionistic matched degree $\tilde{\mu}_{i} \in[a, b] \quad 0 \leq a \leq 1$ $0 \leq b \leq 1 \quad a+b \leq 1$ to the model $i$ is the $i$ th output of 
intuitionistic inference system. According to the expressions (2), we can get the truth-value $\hat{\mu}_{i}$ of $\tilde{\mu}_{i}$ :

$$
\widehat{\mu}_{i}=a+\frac{1}{2} b
$$

In order to be convenient to get the overall estimation of tracking target, the intuitionistic matched degree needs to be normalized as following:

$$
\mu_{i}=\frac{\widehat{\mu}_{i}}{\sum_{N} \widehat{\mu}_{i}}, \sum_{N} \mu_{i}=1
$$

Where $\mu_{i}$ is matched degree of model $i$ to the moving model of the target.

\section{Estimation combination}

According to the results of condition filtering of model $i$ and the matched degree $\mu_{i}$, the overall estimation of maneuvering target is calculated as following:

$$
\begin{gathered}
\hat{X}(k \mid k)=\sum_{i=1}^{N} \hat{X}_{i}(k \mid k) \mu_{i}(k) \\
P(k \mid k)=\sum_{i=1}^{N} \mu_{i}(k)\left\{\begin{array}{l}
P_{i}(k \mid k)-\left[\hat{X}_{i}(k \mid k)-X(k \mid k)\right] \\
\times\left[\hat{X}_{i}(k \mid k)-X(k \mid k)\right]^{T}
\end{array}\right\}
\end{gathered}
$$

\section{EXAMPLE}

The performance of IFMMTA is test with the problem of tracking an aircraft moving in the horizontal plane, described by a coordinated turn model. It was also shown in the references [3][9][10].

The simulation scenario of maneuvering target is designed as following: a non-maneuvering flight during scan 1 to 30 with $300 \mathrm{~m} / \mathrm{s}$; a $180^{\circ}$ turn, lasting from scan 31 50 with rate $3.74^{\circ} / \mathrm{s}$; a non-maneuvering flight during scan 51-70 and then a $-180^{\circ}$ turn with turn rate-3.74/s, lasting from scan 71-95,finally a non-maneuvering flight to the end of scan 100. The target initial state in Cartesian coordinates was:

[0 km $259.8 \mathrm{~m} / \mathrm{s} 0 \mathrm{~km} 150 \mathrm{~m} / \mathrm{s}]$.

The others parameter of simulation scenarios is defined as reference[3]. The performance of IFMMTA and FMMTA is tested by 100times Mont Carlo simulation. The results are shown as figure 1 and figure 2 .

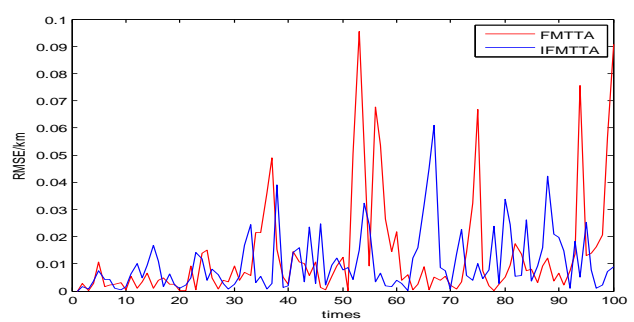

Figure 1. position error of $\mathrm{x}$ (RMS)

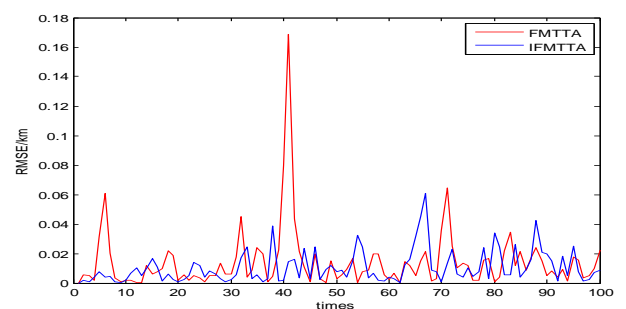

Figure 2. position error of $y($ RMS $)$

With the comparison between FMMTA and IFMMTA from figure 1 and figure 2, we know that IFMMTA has almost the same performance as or better than that of FMMTA if the intuitionistic fuzzy inference system is designed well when tracking maneuvering target. But designed intuitionistic fuzzy inference system is more difficulty than fuzzy inference system.

\section{CONCLUSIONS}

Based on the intuitionistic fuzzy logic inference, IFMMTA is designed in the paper for the un-matching problem between the model and the moving mode due to the target maneuvering. The algorithm solves the confliction between stability and accuracy well by intuitionistic fuzzy logic inference and parallel filtering. The work maybe provide reference for the maneuvering target tracking algorithm designing.

\section{REFERENCES}

[1] Bar- Shalom Y.Extension of the probabilistic data association filter in multi- target racking $[\mathrm{C}] / /$ Proceedings of the 5th Symp on Nonlinear Estimation, 1974: 16- 21.

[2] Pek H F, Gee W N. Combining IMM Method with Particle Filters for 3D Maneuvering Target Tracking[C]//Proc. of the 10th International Conference on Information Fusion. Singapore: [s. n.], 2007.

[3] Zuo Dongguang. Fuzzy Multiple Model Tracking Algorithm for Manoeuvring Target[J] JOURNAL OF XI'AN JIAO TONG UNIVERSIT, vol. 36, no. 2, pp.1241-1246,2006.

[4] Atanassov K. Intuitionistic fuzzy sets [J]. Fuzzy Sets and Systems, vol. 20, no. 1, pp.87-96,March 1986.

[5] Lei Ying-jie. Intuitionistic Fuzzy Conditional Reasoning with the Confidence Spreading[J]. Journal of Electronics \& Information Technology,vol. 28, no. 10, pp.1790-1793, March 2006.

[6] Bustince H. Application to approximate reasoning based on intervalvalued fuzzy sets [J]. International Journal of Approximate Reasoning, vol. 23, no. 2, pp.137-206,2000.

[7] Lei Ying-jie, Wang Bao-shu. On the equivalent mapping between extensions of fuzzy set theory [J]. Systems Engineering and Electronics, vol. 26, no. 11, pp.1414-1418,2004.

[8] Lei Ying-jie, Wang Bao-shu. On the semantic operators for intuitionistic fuzzy logic [J]. Computer Science, vol. 31, no. 11, pp.46, March 2004.

[9] Kim B D, Lee J S. IMM Algorithm Based on the Analytic Solution of Steady State Kalman Filter for Radar Target Tracking[C]//Proc. of the IEEE International on Radar Conference. Seoul, Korea: 2005.

[10] ZHANG Y SH, JI CH X. A fuzzy interacting multiple model algorithm based on current statistical model [J].Fire Control\& Command Concrol, vol. 28, no. 1, pp.51-55,2003. 